\title{
Tanshinone IIA Attenuates Inflammatory Responses by Downregulating MicroRNA-23b, Upregulating TRIB1 and Inactivation of the TLR4/NF-KB Signaling Pathway in THP-1 Macrophages
}

\section{Wei Ji}

Jilin Provincial Avant-garde Hospital

\section{Gengyao Li}

Jilin Provincial Avant-garde Hospital

Yuqian Liu

Jilin Provincial Avant-garde Hospital

Xiaofeng Yan ( $\nabla$ xiaofengY120802@163.com)

Heilongjiang Provincial Avant-garde Hospital https://orcid.org/0000-0002-5113-0935

\section{Research article}

Keywords: Atherosclerosis, Tanshinone IIA, microRNA-23b, TRIB1, TLR4/NF-kB pathway

Posted Date: April 30th, 2020

DOI: https://doi.org/10.21203/rs.3.rs-23071/v1

License: (c) (1) This work is licensed under a Creative Commons Attribution 4.0 International License.

Read Full License 


\section{Abstract}

Background: Atherosclerosis is a kind of chronic cardiovascular disease, and Tanshinone IIA (Tan) is a naturally derived anti-inflammatory compound. In this study, we aim to explore the biological role of Tan and regulatory mechanism in atherosclerosis.

Methods: Firstly, the model of atherosclerosis mice was constructed. The area of atherosclerosis, the level of blood lipid and the content of inflammatory factors were measured in mice treated with Tan. After that, the differentially expressed microRNAs (miRNAs) were obtained by microarray analysis of mice plasma. The effect of miR-23b on cell migration ability, adhesion ability and inflammatory factor level of macrophages treated with Tan. After that, we determined the target mRNA of miR-23b through the bioinformatics analysis and the dual luciferase reporter gene assay. THP-1 macrophages were treated with miR-23b mimic or si-TRIB1 to detect the activity of the TLR4/NF-KB signaling pathway via immunohistochemistry.

Results: Tan injection significantly improved atherosclerosis inflammation in mice, especially in mice treated with $90 \mathrm{mg} / \mathrm{kg}$ dose. miR-23b was obtained by differential screening, which can significantly inhibit the drug effect of Tan, and aggravate the occurrence of atherosclerosis. miR-23b targeted TRIB1, the decrease of TRIB1 also inhibited the drug effect of Tan. The up regulation of miR-23b enhanced the activity of TLR4/NF-KB pathway, and TLR4/NF-KB pathway inhibited the drug effect of Tan on the inflammatory response.

Conclusion: Overall, Tan could protect macrophage against inflammation via inactivating TLR4/NF-KB pathway by down-regulating miR-23b and upregulating TRIB1, providing a novel theoretical foundation for treatment of atherosclerosis.

\section{Background}

Atherosclerosis, a chronic and inflammatory vascular disorder, is one of the most prevalent reasons of cardiovascular diseases (CVD) and a dominant mortality element universally [1]. The pathophysiology of atherosclerosis is firmly connected with the dysregulation of inflammation and immunity [2]. Macrophages play an essential part throughout all phases of atherosclerotic progress, including enlistment and differentiation of immunocytes and dissemination of the inflammatory environment via the emission of pro-inflammatory cytokines [3]. Thus, to examine the practical features of macrophages can help deliver innovative tactics for the management of atherosclerosis and other chronic inflammatory syndromes.

Traditional Chinese medicine shows an imperative influence on the medical treatment of people, which acts as athero-protectors [4]. Tanshinones are the main efficient elements of the Chinese medicinal herb Salvia miltiorrhiza, also known as Danshen, which has been extensively utilized in Asian nations to avert and manage malignant tumors, inflammatory diseases and CVD [5]. Tanshinone IIA (Tan) is one of the greatest bioactive elements among the Tanshinones, owning numerous pharmacological actions such as 
anti-inflammation, anti-oxidation, and anti-atherosclerosis [6]. For instance, Tan considerably exerted an inhibitory impact on the beginning and development of atherosclerosis via restraining the adhesion molecular expression and inflammatory cytokine emission in serum of Apolipoprotein-E knockout (ApoE ${ }^{-/}$ $\left.{ }^{-}\right)$mice [7]. Moreover, outcomes of Yan et al propose that Tan was a latent medical agent that might possess the capability to block atherosclerosis connected with anti-oxidative and anti-inflammatory influences [8]. However, the mechanism of Tan in the management of atherosclerotic disorders is not well-defined to date.

microRNAs (miRNAs), a cluster of small noncoding RNAs, partake in CVD through intervening lipid metabolism, cell viability, mitosis and motility, dysregulated proteolysis, and generation of inflammatory mediators [9]. miRNAs have been described to play chief parts in atherosclerosis pathogenesis via managing inflammatory mediators and lipid homeostasis by distinctive signaling pathways in assorted cell categories [10]. MicroRNA-23b (miR-23b) has been proposed to exhibit a momentous function in distinct conditions, including colon, colorectal, and hepatocellular cancer [11-13]. MiR-23b is linked with autoimmune and inflammation disorders, and miR-23b levels in plasma of rheumatoid arthritis patients were extensively higher than that in healthy people [14]. Therefore, we hypothesized Tan regulated atherosclerosis progression by regulating miR-23b and a certain signaling pathway, while the underlying mechanism remains poorly understood. In the present study, we planned to research the influences of Tan on macrophage inflammation, and uncover the mechanism of Tan with the interaction of miR-23b and related signaling pathway.

\section{Methods}

\section{Animal experiments}

Twelve ApoE ${ }^{-/-}$female mice (8 weeks, 18-21 g) and 3 C57BL/6 female mice (8 weeks, 18-21 g) were purchased from Beijing Vital River Experimental Animal Technology Co, Ltd. (Beijing, China). All mice maintained a normal diet and circadian rhythm for one week. Then, the $\mathrm{ApoE}^{-/-}$female mice were fed with high-fat diet (21\% fat and $0.15 \%$ cholesterol). The C57BL/ 6 female mice were fed for normal diet for 12 weeks, and at the last 4 weeks the mice were randomly assigned into 4 groups ( 3 in each group): MOD group (high-fat diet treatment), T10 group (high-fat diet treatment $+10 \mathrm{mg} / \mathrm{kg}$ Tan injection each day), T30 (high-fat diet treatment $+30 \mathrm{mg} / \mathrm{kg}$ Tan injection each day), T90 (high-fat diet treatment $+90 \mathrm{mg} / \mathrm{kg}$ Tan injection each day). Tan solution was purchased form Sigma-Aldrich (Shanghai, China). The C57BL/ 6 female mice with normal diet were as CON group.

Then the mice were intraperitoneally anaesthetized with $75 \mathrm{mg} / \mathrm{kg}$ pentobarbital sodium (Sigma-Aldrich, Shanghai, China). The blood was collected by the 1.0-1.5-mm glass capillary (immersed in $1 \%$ heparin solution) through eyeball insertion. The blood was added with ethylene diamine tetra acetic acid solution (Thermo Fisher Scientific, Rockford, IL, USA) immediately to avoid clotting, and allowed to stand still in an Eppendorf tube (Sangon Bio., Shanghai, China) for $1 \mathrm{~h}$ at room temperature. After that, the blood was centrifugated at $4^{\circ} \mathrm{C}$ at $1200 \mathrm{~g}$ for $10 \mathrm{~min}$ with the supernatant removed, and then store at $-80^{\circ} \mathrm{C}$ for 
subsequent analysis. The mice were injected with pentobarbital sodium at $75 \mathrm{mg} / \mathrm{kg}$ intraperitoneally and moistened with gauze. After anesthesia, the skin of the anterior chest was cut to expose abdominal cavity and chest. A total of $500 \mathrm{~mL}$ phosphate buffer saline (PBS) solution (Thermo Fisher Scientific, Rockford, IL, USA) was pumped into the left ventricle using a DL10-14 peristaltic pump (LEAD FLUID, Baoding, Hebei, China) at $4^{\circ} \mathrm{C}$ until the outflow fluid was clear. The perfusion was lasted for $4 \mathrm{~h}$ from a relatively slow speed to a higher speed until the death of animals, which was confirmed by observing the lack of heartbeat, respiratory arrest, and nerve reflex. After the perfusion, the lungs were cut off. Under the microscope (LIO0S600T, Trilobite, Beijing, China), the aortic trees were separated, the outer membrane fat was removed and the whole aortic tree was separated from the spine and fixed in $4 \%$ paraformaldehyde for subsequent experiments.

\section{Hematoxylin-eosin (HE) staining}

The cross-section tissues of the aorta of the experimental mice were fixed, embedded in paraffin, sliced at $4 \mu \mathrm{m}$, dewaxed in xylene for $5 \mathrm{~min}$, soaked in alcohol of gradient concentration for 2 min respectively. After washed out alcohol, the sections were soaked in hematoxylin dye solution (Leagene, Anhui, China) for $12 \mathrm{~min}$ followed by floated color washing, soaked in $1 \%$ hydrochloric acid alcohol for $10 \mathrm{~s}$, washed with running water for $40 \mathrm{~min}$, and then immersed into eosin solution (Leagene, Anhui, China) for 4 min followed by rinsing. Subsequently, the sections were dehydrated in gradient alcohol, then transferred into xylene I and xylene II for transparency, and then sealed with gum and observed under a microscope.

\section{Plasma lipid determination}

Total cholesterol (TC), high-density lipoprotein (HDL), low density lipoprotein (LDL) and triglyceride (TG) were measured by automatic biochemical analyzer (RX-2000, Tarrytown, NY, USA). TC and TG were detected by enzyme method, and LDL and HDL were detected by turbidimetry.

\section{Enzyme-linked immunosorbent assay (ELISA)}

The contents of necrosis factor (TNF)-a and interleukin (IL)- 6 were determined by Enzyme Immunoassay kits (Enzo Life Sciences, Shanghai, China). The specific antibody globulin was diluted to $10 \mathrm{ug} / \mathrm{mL}$, which was added to the plate $(0.3 \mathrm{~mL} /$ well $)$ and incubated at $4^{\circ} \mathrm{C}$ overnight. The cells were incubated with $0.2 \mathrm{~mL}$ diluted plasma at $37^{\circ} \mathrm{C}$ for $1 \mathrm{~h}, 0.2 \mathrm{~mL}$ diluted enzyme labeled antibody solution at $37^{\circ} \mathrm{C}$ for $1 \mathrm{~h}$ and $0.2 \mathrm{~mL}$ substrate at room temperature for $30 \mathrm{~min}$. Finally, $0.05 \mathrm{~mL} \mathrm{H}_{2} \mathrm{SO}_{4}$ was added to each well to terminate the reaction. The enzyme marker $(\mathrm{OPD}=492 \mathrm{~nm})$ was applied to determine optical density (OD) value.

\section{Microarray analysis}

Total RNA $(0.5 \mu \mathrm{g})$ was extracted from mouse aortic plasma. cDNA was synthetized by GeneChip Invitro Tran-scription Express Kit (902789, Thermo Fisher Scientific, Rockford, IL, USA). Then, cDNA fragments were hybridized with miRCURY LNA Array v.16.0 (Axon GenePix, Sunnyvale, CA, USA). The microarray was washed and scanned by GeneChipTM Scanner $30007 \mathrm{G}$ system $(000213$, Thermo Fisher Scientific, Rockford, IL, USA). 


\section{RNA isolation and quantitation}

Total RNA from cardiovascular tissues and THP-1-derived macrophages was isolated by TRIzol. Reverse transcription and amplification of the same amount of RNA into the complementary DNA (CDNA) were conducted according to the manufacturer's instructions PrimeScript ${ }^{\text {TM }}$ RT Master Mix Kit (Takara, Tokyo, Japan). Gene expression was quantified by SYBR®Premix Ex Taq ${ }^{T M} I I$ kit (Takara, Tokyo, Japan). The primers involved in this study were as follows:

miR-23b: Forward, 5'-CAGGCAAGATGCTGTTGCA-3'; Reverse, 5'-GCGAGCACAGAATTAATACGACTC-3';

Tribbles 1 (TRIB1): Forward, 5'-ACGTTGGATGTAGAAGTCCCCTTCCCTTAG-3'; Reverse, 5'ACGTTGGATGGAACAAGGACTTTCGTCCTC-3'.

Reverse transcription quantitative polymerase chain reaction (RT-qPCR) was performed on Mx3005P quantitative PCR system (Stratagene, Shanghai, China) according to the instructions: $95^{\circ} \mathrm{C}$ for $10 \mathrm{~min}, 40$ cycles of $95^{\circ} \mathrm{C}$ for $15 \mathrm{~s}, 55^{\circ} \mathrm{C}$ for $20 \mathrm{~s}$ and $72^{\circ} \mathrm{C}$ for $30 \mathrm{~s}$. $\beta$-actin was used as an internal control. All samples were analyzed in triplicate. The relative expression levels of RNA were calculated using $2^{-\Delta \Delta C t}$ method.

\section{Cell culture and treatment}

Human monocytes THP-1 cells were obtained from the cultured specimens (American Tissue Culture Collection, Manassas, VA) and grown in RPMI-1640 medium containing 10\% fetal bovine serum (FBS) at $37^{\circ} \mathrm{C}$ and an atmosphere of $\angle 5 \% \mathrm{CO}_{2}$. THP-1 cells were differentiated into macrophages by incubating with $150 \mathrm{ng} / \mathrm{mL}$ phorbol 12-myristate 13-acetate for $48 \mathrm{~h}$. After washed by PBS, the cells were observed under a microscope (LIOOS600T, Trilobite, Beijing, China). THP-1-derived macrophages were stimulated with $10 \mu \mathrm{mol} / \mathrm{L}$ Tan for $1 \mathrm{~h}$ at $37^{\circ} \mathrm{C}$ and $5 \% \mathrm{CO}_{2}$. Finally, the macrophages were washed by PBS after Tan absorption and used in further experiments.

Twenty-four h before transfection, the differentiated macrophages were cultured in antibiotics-free RPMI1640 with $10 \%$ PBS and without and placed in a 24-well plate. Before transfection, the culture medium was changed to Opti-MEM I reducing serum medium (Grand Island, NY, USA). Using Lipofectamine 2000 (Invitrogen, Carlsbad, USA), the miR-23b mimic, empty plasmid, or small interfering RNA-targeting TRIB1 (si-TRIB1) were transfected to the macrophages for $6 \mathrm{~h}$. The cell transfection efficiency was observed under a fluorescence microscope (Olympus Corporation, Tokyo, Japan).

\section{Transwell assay}

After adding Matrigel (BD Biosciences, Franklin lakes, NJ, USA) to the apical chamber for 30 min under specific pathogen free condition, $30 \mu \mathrm{L}$ RPMI-1640 medium was added to the apical chamber and placed in $\mathrm{CO}_{2}$ incubator for standby. The cells were digested, centrifuged, resuspended without serum and diluted to $5 \times 10^{5}$ cells $/ \mathrm{mL}$. then, $500 \mu \mathrm{L}$ RPMI 1640 medium containing $10 \%$ FBS was added into the basolateral chamber of Transwell while $200 \mu \mathrm{L}$ cell suspension was added into the upper chamber, and 
then the Transwell plate was cultured in $37^{\circ} \mathrm{C}, 5 \% \mathrm{CO}_{2}$ incubator for $48 \mathrm{~h}$. The chamber was taken out, and the culture medium was washed out with PBS. After that, the cells were stained with crystal violet for 10 min with the floating color and suspended cells washed off. Finally, the invasive cells were observed under the microscope (LIOOS600T, Trilobite, Beijing, China) and observed and the number of cells was counted.

\section{Cell adhesion assay}

Ten $\mu \mathrm{g} / \mathrm{mL}$ of fibronectin (Abcam Inc., Cambridge, MA, USA) at $70 \mu \mathrm{L} /$ well was injected into the 96 -well plates and incubated overnight at $4{ }^{\circ} \mathrm{C}$. After 3 times of PBS cleaning, the cells were sealed with $1 \%$ bovine serum albumin (Sigma Aldrich, Shanghai, China) at $37^{\circ} \mathrm{C}$ for $1 \mathrm{~h}$. After PBS cleaning 3 times, the cells at the logarithmic growth phase were digested, suspended in the serum-free medium. The cell concentration was adjusted at $5 \times 10^{4}$ cells $/ \mathrm{mL}$ and $100 \mu \mathrm{L} /$ well. Each group was established three wells. The cells were incubated at $37^{\circ} \mathrm{C}$ for $1 \mathrm{~h}$, the suspension was washed off by PBS. The cells were fixed by formaldehyde and stained by crystal violet followed by PBS washing. Under the microscope, 5 visual fields were selected for photographing and counting. The average value was taken.

\section{Dual luciferase reporter gene assay}

The 3'UTR binding sequences of miR-23b were predicted by online prediction software Starbase (http://starbase.sysu.edu.cn/). The 3'UTR of wild type (WT) and mutant (MUT) of miR-23b was synthesized by Sangon Biotechnology Co., Ltd. (Shanghai, China) and inserted into pMIR-REPORTTM (Thermo Fisher Scientific Inc., Waltham, MA, USA) luciferase reporter vector [15]. Using the Lipofectamine 3000 transfection Kit (Invitrogen, Carlsbad, CA, USA), WT/MUT plasmids and miR-23b were cotransfected into THP-1 cells. After $24 \mathrm{~h}$, the cells were lysed. Luciferase activity intensity (intensity = RLU1/RLU2, RLU1 is the luciferase activity of firefly, RLU1 is the luciferase activity of renilla) was detected by dual luciferase reporter assay system (Promega Corporation, WI, USA) [15].

\section{Immunohistochemistry}

After dewaxing and hydration, the sections of mouse aorta were infiltrated with sealing permeable liquid (Solarbio, Beijing, China) for $30 \mathrm{~min}$. The sections were added into sodium citrate buffer solution (50 x, Solarbio, Beijing, China) and heat for $6 \mathrm{~min} \times 4$ times, and dropped with 5\% sheep serum (Solarbio, Beijing, China) and allowed to stand still for $30 \mathrm{~min}$ at room temperature. The serum was removed and the cells were incubated with the primary antibodies against nuclear factor kappa B (NF-kB) (ab16502) and Toll-like receptor 4 (TLR4) (1:500, ab13556) at room temperature for $1 \mathrm{~h}$ and $4^{\circ} \mathrm{C}$ overnight. Then, the cells were washed by PBS and incubated with the secondary antibody $\left(1: 50000\right.$, ab205718) at $37^{\circ} \mathrm{C}$ for $30 \mathrm{~min}$. subsequently, the cells were incubated with streptavidin peroxidase (Solarbio, Beijing, China) at $37^{\circ} \mathrm{C}$ for 30 min. finally, the cells were added with Diaminobenzidine (20x, Solarbio, Beijing, China) for 5 min followed by 20-s hematoxylin staining. The cells were dehydrated, sealed in gum and observed under a microscope.

\section{Statistical analysis}


All statistical analyses were performed using SPSS 21.0 (IBM Corp. Armonk, NY, USA). Data were in normal distribution according to Kolmogorov-Smimov method and described as mean \pm standard deviation. Differences between two groups were analyzed by $t$ test. Differences among multiple groups were analyzed using one-way analysis of variance (ANOVA) or two-way ANOVA. Tukey's multiple comparisons test was used for the pairwise comparison after ANOVA analysis. $p$ was obtained by twotailed test and $p<0.05$ was considered statistically significant.

\section{Results}

\section{Atherosclerosis mice model is established successfully}

First of all, we identified the atherosclerosis model by histological experiment. Compared with the CON group, the MOD group showed larger atheromatous plaque area (Fig. 1A), increased contents of TC, LDL, HDL and TG in plasma (Fig. 1B), and increased contents of TNF-a and IL-6 (Fig. 1C).

\section{Tan relieves macrophage inflammation of atherosclerosis}

We detected the relative atherosclerotic area of the MOD, T10, T30 and T90 groups, and found that with the increasing injection amount of Tan, the relative atherosclerotic area decreased (Fig. 2A). Similarly, with the increasing injection amount of Tan, the contents of TC, LDL, HDL and TG in plasma decreased (Fig. 2B). With the increasing injection amount of Tan, the content of TNF-a and IL-6 decreased detected by ELISA (Fig. 2C).

\section{miR-23b is dysregulated in macrophage of atherosclerosis after Tan treatment}

miR-23b in total RNA extracted from the MOD, T10, T30 and T90 groups was obtained by microarray analysis (Fig. 3A). The results of microarray analysis were verified by RT-qPCR (Fig. 3B).

\section{Overexpression of miR-23b enhances macrophage inflammation of atherosclerosis}

Firstly, it was confirmed that miR-23b mimic model was constructed successfully (Fig. 4A). After overexpression of miR-23b, migration ability (Fig. 4B), adhesion ability (Fig. 4C), TNF-a and IL-6 contents increased in macrophage (Fig. 4D).

\section{miR-23b targets TRIB1 to regulate the TLR4/NF-KB signaling pathway}


Starbase analysis showed that there were nucleotide binding sites between miR-23b and TRIB1, and dual luciferase reporter gene assay showed that the target gene of miR-23b is TRIB1 (Fig. 5A). The content of TRIB1 in the CON, MOD, T10, T30 and T90 groups was detected. With the increasing injection amount of Tan, the content of TRIB1 increased gradually (Fig. 5B). At the same time, compared with miR-23b control treatment, miR-23b mimic treatment decreased the content of TRIB1 significantly (Fig. 5C). With the increasing injection amount of Tan, the content of TLR4 and NF-KB decreased (Fig. 5D). Compared with miR-23b control treatment, miR-23b mimic treatment decreased contents of TLR4 and NF-KB (Fig. 5E).

\section{TRIB1 knockdown enhances the macrophage inflammation of atherosclerosis}

Firstly, it was confirmed that si-TRIB1 model was constructed successfully (Fig. 6A). After si-TRIB1 treatment, migration ability (Fig. 6B), adhesion ability (Fig. 6C), TNF- $a$ and IL-6 contents increased in macrophage (Fig. 6D). Besides, TLR4/NF-KB activity was enhanced by si-TRIB1 in macrophage (Fig. 6E).

\section{Discussion}

Atherosclerosis comprises assorted acellular and cellular manners including inflammatory factor generation, macrophage polarization, plaque rupture, and oxidative stress response [16]. Moreover, abundant data exhibit that the high expression and activation of inflammatory cytokines can boost the growth of atherosclerosis [17]. Emerging evidence has indicated that Tan has been generally applied to management of atherosclerosis $[6,18,19]$. Nevertheless, the underlying system needed to be further studied for improved application of Tan in clinical. In our study, we acquired that Tan decreased the inflammatory response by regulating expressions of miR-23b and the TRIB1/TLR4/NF-KB axis.

Our findings revealed that Tan efficiently hindered inflammatory injury through raising cell viability, suppressing apoptosis, and diminished productions of IL-6 and TNF- $\alpha$ in macrophages. Macrophages are involved in complicated environmental settings, such as inflammatory cytokines and lipids, which regulates their practical phenotypes during the development of atherosclerotic plaque [20]. Tan, as a constituent of tanshinones, is involved in THP-1 macrophages by displaying anti-inflammatory action [21]. Moreover, preceding work proposed that Tan inhibited cholesterol enlargement by controlling heme oxygenase-1 in human macrophages [22]. Analogously, our paper also uncovered the inhibitory impacts of Tan on apoptosis and inflammation in THP-1-derived macrophages. IL-6 and TNF-a have been recognized as pro-atherogenic cytokines which can be released by natural killer cells, lymphocytes, vascular smooth muscle cells and macrophages [23]. Consistently, earlier reports revealed that Tan hindered the pro-inflammatory cytokine production in RAW264.7 murine macrophage cells and the serum of $\mathrm{ApoE}^{-/-}$mice $[24,25]$. Also, Ma et al. proved that Tan restrained the levels of IL-1 $\beta$ and TNF-a in lipopolysaccharide-induced THP-1 macrophages [21]. Moreover, Tan had varied biological capabilities, such as anti-oxidation, anti-apoptosis, anti-inflammation, anti-cancer, improving microcirculation, and 
anti-myocardial ischemia [26]. Yet, the molecular system allows Tan connected with atheroprotection is inadequately identified [4].

We also showed that atherosclerosis could provoke the remarkable upregulation of miR-23b and miR-23b overexpression eliminated the inhibitory influence of Tan on pro-inflammatory cytokine secretion in macrophages. miR-23b is encoded by the chromosomal region $9 q 22.32$, which belongs to the miR$23 \mathrm{~b} / 27 \mathrm{~b} / 24-1$ family cluster and serves as a pleiotropic mediator in numerous processes physiologically and pathologically [13]. MiR-23b-3p is upregulated in type 2 diabetes patients with obesity, which is associated with fibrosis through matrix metalloproteinases [27]. The effect of miR-23b on inflammation shows diverse presentation in different disease. For instance, a previous study of Hu et al illustrated that miR-23b played a protective role in intracerebral hemorrhage via inhibiting neuroinflammation [28]. However, a study of $\mathrm{He}$ et al revealed that downregulation of miR-23b could relieve oxidized-LDL-induced inflammation in macrophages [29]. In our study, Tan might relieve inflammatory response in macrophages through down-regulating miR-23b. Tan could act as inflammation inhibitor by controlling a series of cytokine secretion and miRNA expression in lipopolysaccharide-induced cells [30]. For example, Tan attenuates hypoxia-induced PC-12 cell damage through preventing miR-28 up-regulation [31]. Tan prevented pro-inflammatory cytokine secretion induced by oxidized-LDL via inhibiting miR-33 overexpression in THP-1 macrophages [16].

Finally, we discovered that Tan prevents inflammation of macrophages by upregulating TRIB1 and inactivating the TLR4/NF-KB signaling pathway, and activation of the pathway was modulated by miR23b. Single nucleotide polymorphisms of TRIB1 are associated with TC, triglyceride, apolipoprotein A1, LDL, and HDL levels, which contribute to the risk of ischemic stroke and coronary heart disease [32]. Moreover, TRIB1 overexpression accelerates macrophage cholesterol efflux to apolipoprotein A-I through blocking lipid accumulation in oxidized-LDL-treated THP-1 macrophages [33]. TRIB1 is also involved in the activities of the NF-KB signaling pathway, which participates in cell proliferation and inflammatory response in prostate cancer [34]. The TLR4/NF-KB signaling pathway has noteworthy functions in the inflammation and stress response, which has been recently recommended to be firmly connected with human atherosclerosis [35]. MiR-23b enhances oxidized-LDL-stimulated inflammatory reaction of macrophages and contributes to atherosclerosis through the A20/NF-KB signaling pathway at high cardiovascular risk in the Mongolian [36]. MiR-23b could also attenuate apoptosis of BV-2 cells by modifying the NF-KB signaling pathway and could perform as an innovative approach for the management of spinal cord injury [37]. Tan could alleviate susceptible atherosclerotic plaque in $\mathrm{ApoE}^{-/-}$ mice, and the immune-regulating and anti-inflammatory impact may be accomplished via the TLR4/myeloid differentiation factor88 (MyD88)/NF-KB signaling pathway [38]. The inhibitory impact of Tan on the MMP-9 level and inflammation generated by oxidized-LDL via the inactivation of TLR4/NF-KB signaling pathway in RAW 264.7 macrophages [39].

\section{Conclusions}


Taken together, our data revealed that Tan alleviated inflammatory injury by targeting miR-23b and regulating the TRIB1/TLR4/NF-KB axis in human macrophages (Fig. 7). The findings in this current study proposed a novel miRNA target of Tan for atherosclerosis treatment and developing our understanding on the Tan etiology.

\section{Abbreviations}

Tan: Tanshinone IIA; miRNAs: microRNAs; CVD: cardiovascular diseases; TRIB1: Tribbles 1; ApoE-/-: Apolipoprotein-E knockout; PBS: phosphate buffer saline; HE: hematoxylin-eosin; TC:total cholesterol; HDL: high-density lipoprotein; LDL: Iow density lipoprotein; TG: triglyceride; ELISA: enzyme-linked immunosorbent assay; TNF-a: necrosis factor; IL-6: interleukin; OD: optical density; cDNA: complementary DNA; RT-qPCR: reverse transcription quantitative polymerase chain reaction; FBS: si-TRIB1: fetal bovine serum; small interfering RNA-targeting TRIB1; WT: wild type; MUT: mutant; NF-kB:nuclear factor kappa B; TLR4: Toll-like receptor 4; ANOVA: analysis of variance.

\section{Declarations}

\section{Ethics approval and consent to participate}

All protocols were approved by the Animal Ethics Committee of Jilin Provincial Avant-garde Hospital.

\section{Consent for publication}

Not applicable.

\section{Availability of data and materials}

The datasets used and/or analyzed during the current study are available from the corresponding author on reasonable request.

\section{Competing interests}

The authors report no conflicts.

\section{Funding}

Not applicable.

\section{Authors' contributions}


WJ conceived the study and participated in its design and coordination. GYL performed all experiments. YQL and XFY collected tissue samples and clinical data. WJ and XFY analyzed and interpreted the data. The draft was improved through discussion and editing by all the authors who read and approved the final manuscript.

\section{Acknowledgements}

Not applicable.

\section{References}

1. Pakzad B, Rajae E, Shahrabi S, Mansournezhad S, Davari N, Azizidoost S, Saki N. T-Cell Molecular Modulation Responses in Atherosclerosis Anergy. Lab Med 2020.

2. Yan Y, Song D, Wu J, Wang J. Long Non-Coding RNAs Link Oxidized Low-Density Lipoprotein With the Inflammatory Response of Macrophages in Atherogenesis. Front Immunol 2020, 11:24.

3. Kavurma MM, Rayner KJ, Karunakaran D. The walking dead: macrophage inflammation and death in atherosclerosis. Curr Opin Lipidol 2017, 28(2):91-98.

4. Yuan L, Li Q, Zhang Z, Liu Q, Wang X, Fan L. Tanshinone IIA inhibits the adipogenesis and inflammatory response in ox-LDL-challenged human monocyte-derived macrophages via regulating miR-130b/WNT5A. J Cell Biochem 2020, 121(2):1400-1408.

5. Ren J, Fu L, Nile SH, Zhang J, Kai G. Salvia miltiorrhiza in Treating Cardiovascular Diseases: A Review on Its Pharmacological and Clinical Applications. Front Pharmacol 2019, 10:753.

6. Zhu J, Xu Y, Ren G, Hu X, Wang C, Yang Z, Li Z, Mao W, Lu D. Tanshinone IIA Sodium sulfonate regulates antioxidant system, inflammation, and endothelial dysfunction in atherosclerosis by downregulation of CLIC1. Eur J Pharmacol 2017, 815:427-436.

7. Wang B, Ge Z, Cheng Z, Zhao Z. Tanshinone IIA suppresses the progression of atherosclerosis by inhibiting the apoptosis of vascular smooth muscle cells and the proliferation and migration of macrophages induced by ox-LDL. Biol Open 2017, 6(4):489-495.

8. Xuan Y, Gao Y, Huang H, Wang X, Cai Y, Luan QX. Tanshinone IIA Attenuates Atherosclerosis in Apolipoprotein E Knockout Mice Infected with Porphyromonas gingivalis. Inflammation 2017, 40(5):1631-1642.

9. Johnson JL. Elucidating the contributory role of microRNA to cardiovascular diseases (a review). Vascul Pharmacol 2019, 114:31-48.

10. Donaldson CJ, Lao KH, Zeng L. The salient role of microRNAs in atherogenesis. J Mol Cell Cardiol 2018, 122:98-113.

11. Gasiule S, Dreize N, Kaupinis A, Razanskas R, Ciupas L, Stankevicius V, Kapustina Z, Laurinavicius A, Valius M, Vilkaitis G. Molecular Insights into miRNA-Driven Resistance to 5-Fluorouracil and 
Oxaliplatin Chemotherapy: miR-23b Modulates the Epithelial-Mesenchymal Transition of Colorectal Cancer Cells. J Clin Med 2019, 8(12).

12. Liu Y, Tan J, Ou S, Chen J, Chen L. Adipose-derived exosomes deliver miR-23a/b to regulate tumor growth in hepatocellular cancer by targeting the VHL/HIF axis. J Physiol Biochem 2019, 75(3):391401.

13. Nishida K, Kuwano Y, Rokutan K. The MicroRNA-23b/27b/24 Cluster Facilitates Colon Cancer Cell Migration by Targeting FOXP2. Cancers (Basel) 2020, 12(1).

14. Zhang W, Lu F, Xie Y, Lin Y, Zhao T, Tao S, Lai Z, Wei N, Yang R, Shao Y, et al. miR-23b Negatively Regulates Sepsis-Induced Inflammatory Responses by Targeting ADAM10 in Human THP-1 Monocytes. Mediators Inflamm 2019, 2019:5306541.

15. Jiang C, Zhu W, Xu J, Wang B, Hou W, Zhang R, Zhong N, Ning Q, Han Y, Yu H, et al. MicroRNA-26a negatively regulates toll-like receptor 3 expression of rat macrophages and ameliorates pristane induced arthritis in rats. Arthritis Res Ther 2014, 16(1):R9.

16. Yang C, Lei X, Li J. Tanshinone IIA reduces oxidized low-density lipoprotein-induced inflammatory responses by downregulating microRNA-33 in THP-1 macrophages. Int J Clin Exp Pathol 2019, 12(10):3791-3798.

17. Wolf MP, Hunziker P. Atherosclerosis: Insights into Vascular Pathobiology and Outlook to Novel Treatments. J Cardiovasc Transl Res 2020.

18. Li Y, Guo Y, Chen Y, Wang Y, You Y, Yang Q, Weng X, Li Q, Zhu X, Zhou B, et al. Establishment of an interleukin-1 beta-induced inflammation-activated endothelial cell-smooth muscle cell-mononuclear cell co-culture model and evaluation of the anti-inflammatory effects of tanshinone IIA on atherosclerosis. Mol Med Rep 2015, 12(2):1665-1676.

19. Wang J, He X, Chen W, Zhang N, Guo J, Liu J, Zhang L, Sun H, Jia L, Yang G. Tanshinone IIA protects mice against atherosclerotic injury by activating the TGF-beta/PI3K/Akt/eNOS pathway. Coron Artery Dis 2019.

20. Chen W, Li X, Guo S, Song N, Wang J, Jia L, Zhu A. Tanshinone IIA harmonizes the crosstalk of autophagy and polarization in macrophages via miR-375/KLF4 pathway to attenuate atherosclerosis. Int Immunopharmacol 2019, 70:486-497.

21. Ma S, Zhang D, Lou H, Sun L, Ji J. Evaluation of the anti-inflammatory activities of tanshinones isolated from Salvia miltiorrhiza var. alba roots in THP-1 macrophages. J Ethnopharmacol 2016, 188:193-199.

22. Liu Z, Wang J, Huang E, Gao S, Li H, Lu J, Tian K, Little PJ, Shen X, Xu S, et al. Tanshinone IIA suppresses cholesterol accumulation in human macrophages: role of heme oxygenase-1. J Lipid Res 2014, 55(2):201-213.

23. Liberale L, Carbone F, Montecucco F, Sahebkar A. Statins reduce vascular inflammation in atherogenesis: A review of underlying molecular mechanisms. Int J Biochem Cell Biol 2020, 122:105735. 
24. Gao S, Wang Y, Li D, Guo Y, Zhu M, Xu S, Mao J, Fan G. TanshinonellA Alleviates Inflammatory Response and Directs Macrophage Polarization in Lipopolysaccharide-Stimulated RAW264.7 Cells. Inflammation 2019, 42(1):264-275.

25. Liu X, Guo CY, Ma XJ, Wu CF, Zhang Y, Sun MY, Pan YT, Yin HJ. Anti-inflammatory effects of tanshinone IIA on atherosclerostic vessels of ovariectomized ApoE mice are mediated by estrogen receptor activation and through the ERK signaling pathway. Cell Physiol Biochem 2015, 35(5):17441755.

26. Liu X, Meng J. Tanshinone IIA ameliorates lipopolysaccharide-induced inflammatory response in bronchial epithelium cell line BEAS-2B by down-regulating miR-27a. Biomed Pharmacother 2018, 104:158-164.

27. Brovkina O, Nikitin A, Khodyrev D, Shestakova E, Sklyanik I, Panevina A, Stafeev I, Menshikov M, Kobelyatskaya A, Yurasov A, et al. Role of MicroRNAs in the Regulation of Subcutaneous White Adipose Tissue in Individuals With Obesity and Without Type 2 Diabetes. Front Endocrinol (Lausanne) 2019, 10:840.

28. Hu L, Zhang H, Wang B, Ao Q, Shi J, He Z. MicroRNA-23b alleviates neuroinflammation and brain injury in intracerebral hemorrhage by targeting inositol polyphosphate multikinase. Int Immunopharmacol 2019, 76:105887.

29. He L, Zhao X, He L. LINC01140 Alleviates the Oxidized Low-Density Lipoprotein-Induced Inflammatory Response in Macrophages via Suppressing miR-23b. Inflammation 2020, 43(1):66-73.

30. Fan G, Jiang X, Wu X, Fordjour PA, Miao L, Zhang H, Zhu Y, Gao X. Anti-Inflammatory Activity of Tanshinone IIA in LPS-Stimulated RAW264.7 Macrophages via miRNAs and TLR4-NF-kappaB Pathway. Inflammation 2016, 39(1):375-384.

31. Tang N, Chang J, Zeng Y, Zheng J. Tanshinone IIA protects hypoxia-induced injury by preventing microRNA-28 up-regulation in PC-12 cells. Eur J Pharmacol 2019, 854:265-271.

32. Zhang QH, Yin RX, Chen WX, Cao XL, Wu JZ. TRIB1 and TRPS1 variants, G X G and G x E interactions on serum lipid levels, the risk of coronary heart disease and ischemic stroke. Sci Rep 2019, 9(1):2376.

33. Fu Y, Zhao Y, Huang B. Tribbles homolog 1 enhances cholesterol efflux from oxidized low-density lipoprotein-loaded THP-1 macrophages. Exp Ther Med 2017, 14(1):862-866.

34. Bornigen D, Tyekucheva S, Wang X, Rider JR, Lee GS, Mucci LA, Sweeney C, Huttenhower C. Computational Reconstruction of NFkappaB Pathway Interaction Mechanisms during Prostate Cancer. PLoS Comput Biol 2016, 12(4):e1004820.

35. Zhang X, Xue C, Xu Q, Zhang Y, Li H, Li F, Liu Y, Guo C. Caprylic acid suppresses inflammation via TLR4/NF-kappaB signaling and improves atherosclerosis in ApoE-deficient mice. Nutr Metab (Lond) 2019, 16:40.

36. He LP, Zhao XS, He LP. Abnormally expressed miR-23b in Chinese Mongolian at high cardiovascular risk may contribute to monocyte/macrophage inflammatory reaction in atherosclerosis. Biosci Rep 2018, 38(6). 
37. Zhou X, Chen J, Zhang H, Chen X, Shao G. MicroRNA-23b attenuates the H2O2-induced injury of microglial cells via TAB3/NF-kappaB signaling pathway. Int J Clin Exp Pathol 2018, 11(12):57655773.

38. Chen Z, Gao X, Jiao Y, Qiu Y, Wang A, Yu M, Che F, Li S, Liu J, Li J, et al. Tanshinone IIA Exerts AntiInflammatory and Immune-Regulating Effects on Vulnerable Atherosclerotic Plaque Partially via the TLR4/MyD88/NF-kappaB Signal Pathway. Front Pharmacol 2019, 10:850.

39. Wang N, Zhang X, Ma Z, Niu J, Ma S, Wenjie W, Chen J. Combination of tanshinone IIA and astragaloside IV attenuate atherosclerotic plaque vulnerability in $\mathrm{ApoE}(-/-)$ mice by activating PI3K/AKT signaling and suppressing TRL4/NF-kappaB signaling. Biomed Pharmacother 2020, 123:109729.

\section{Figures}
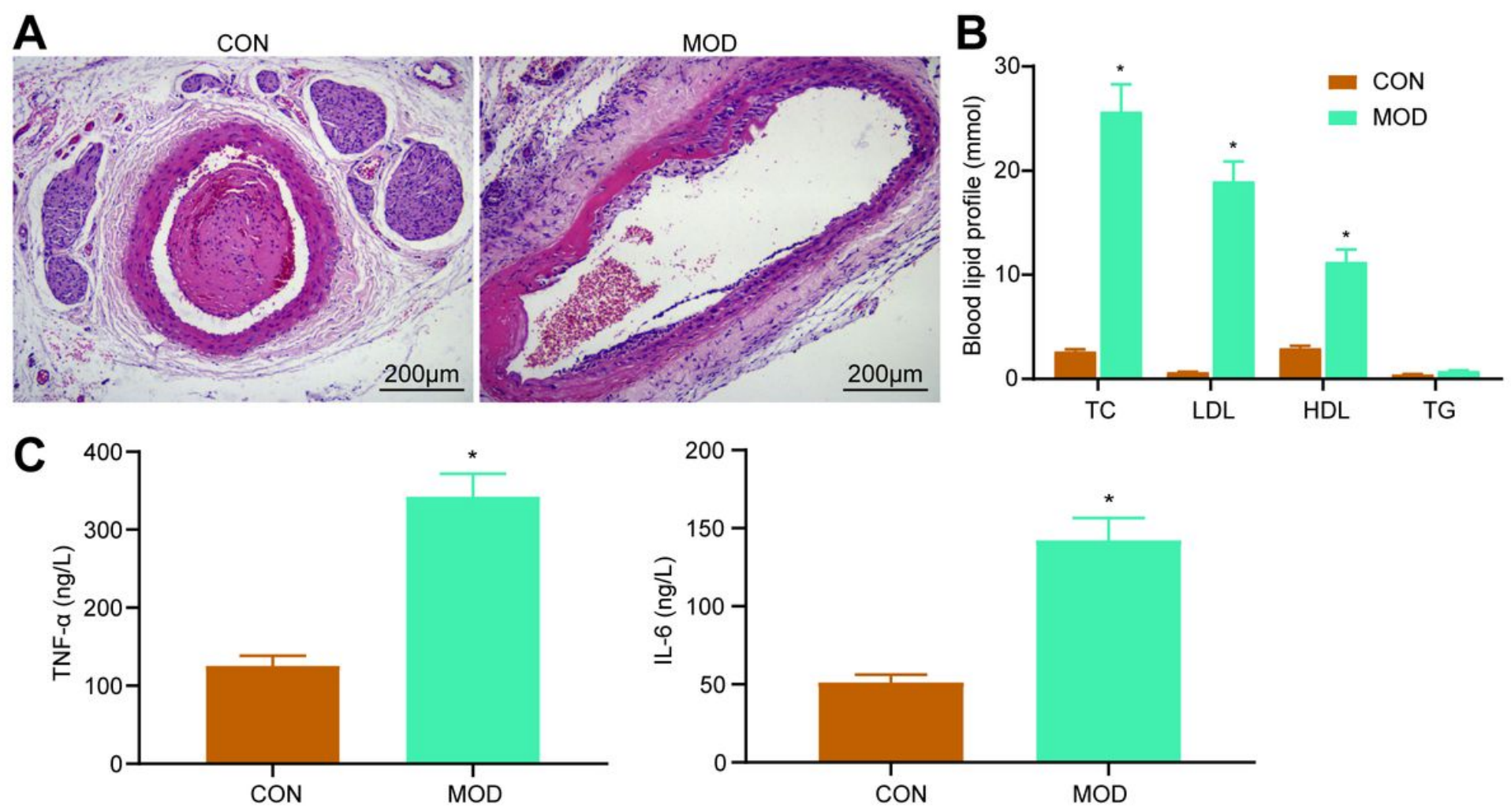

Figure 1

Atherosclerosis mice model determination. A, HE staining for the atherosclerotic area of the cross section of mouse aorta. $B$, contents of TC, LDL, HDL and TG in plasma ( ${ }^{*} p<0.05$ according to the two-way ANOVA). C, TNF- $a$ and IL- 6 levels in mouse aortic plasma detected by ELSA ( ${ }^{\star} p<0.05$ according to the unpaired $t$ test). All experiment was conducted 3 times independently. 

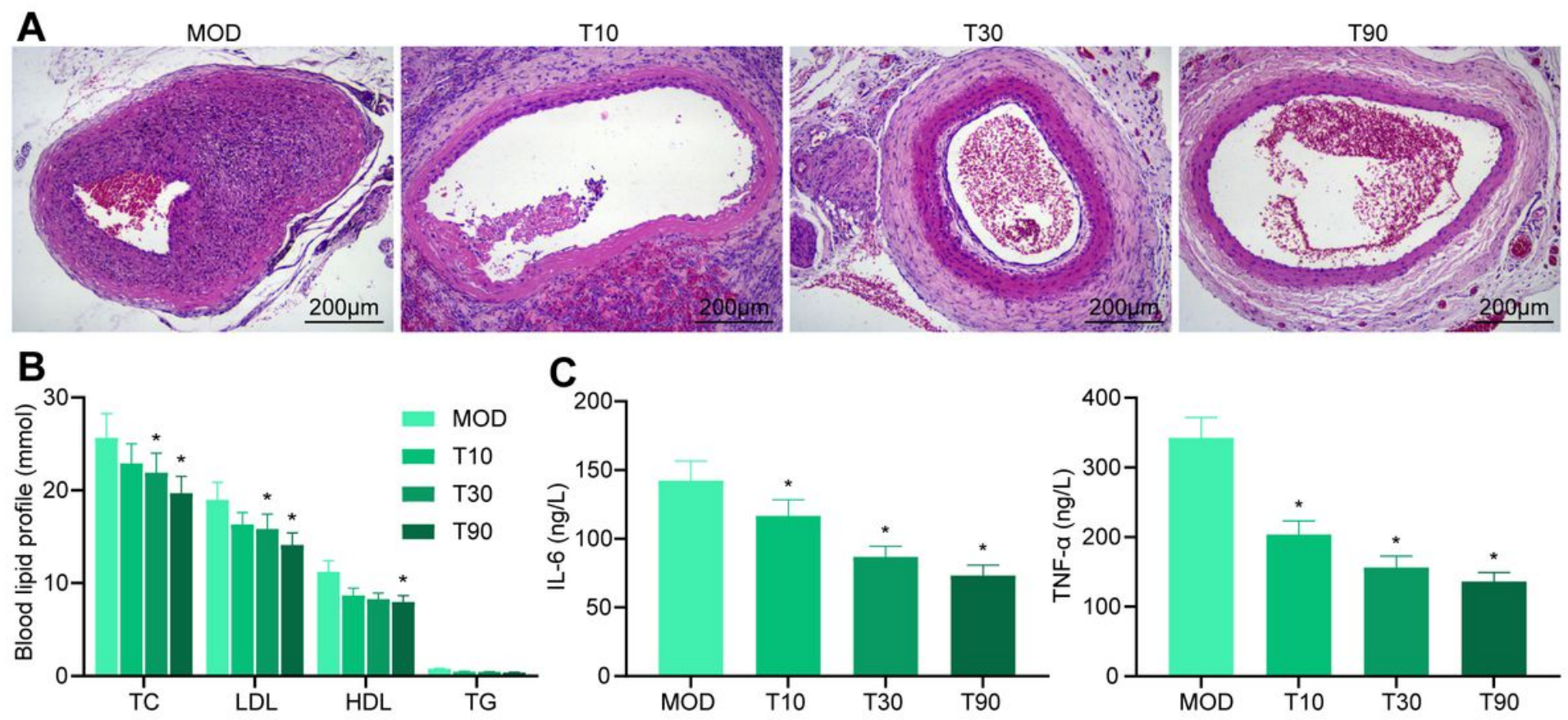

Figure 2

Effect of Tan on atherosclerosis. A, cross-sectional atherosclerotic area of aorta in Tan treated mice by HE staining. $B$, the contents of TC, LDL, HDL and TG in plasma detected by plasma biochemical analysis ( ${ }^{*} p<0.05$ according to the two-way ANOVA). C, TNF- $a$ and IL- 6 levels in mouse aortic plasma detected by ELSA ( ${ }^{*} p<0.05$ according to the one-way ANOVA). All experiment was conducted 3 times independently.

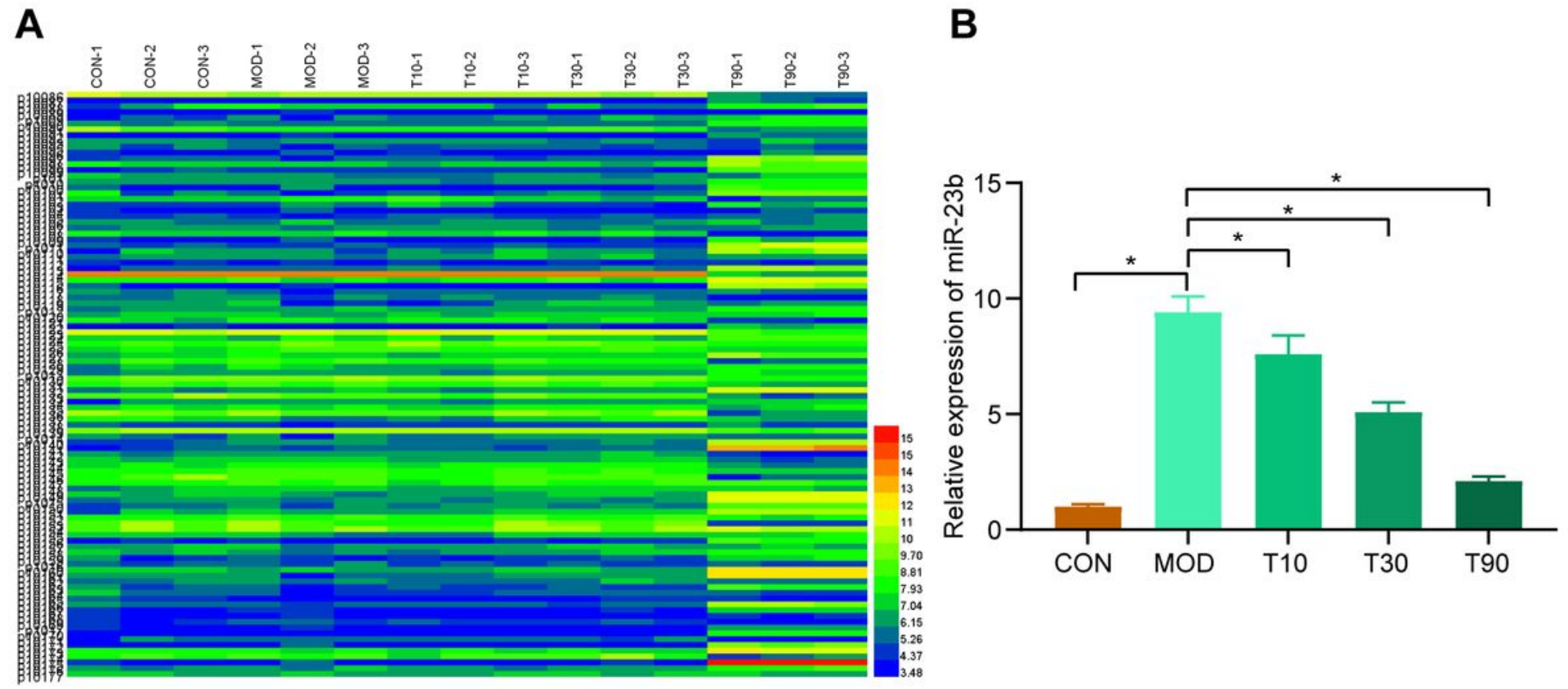

\section{Figure 3}

Differentially expressed miRNA in plasma after atherosclerosis and Tan treatment. A, miRNA expression in plasma after Tan treatment analyzed by microarray analysis. B, miR-23b expression detected by RTqPCR ( ${ }^{*} p<0.05$ according to the one-way ANOVA). All experiment was conducted 3 times independently. 


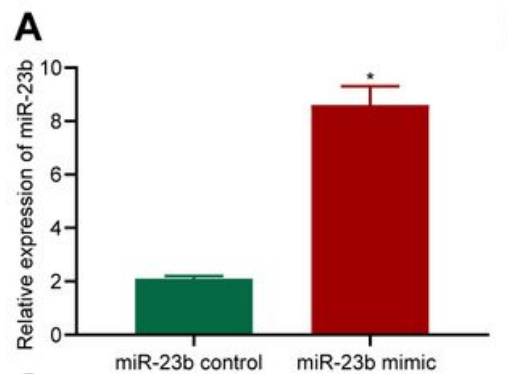

C
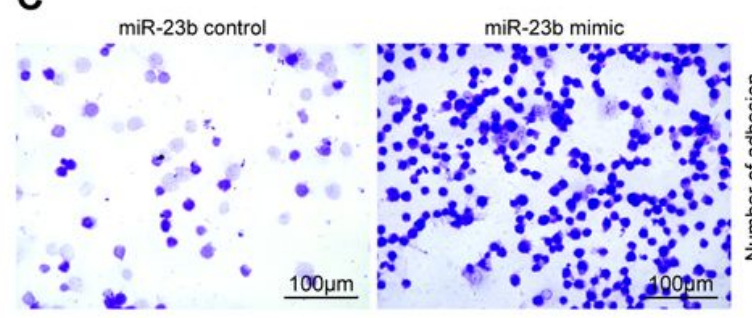

B miR-23b control
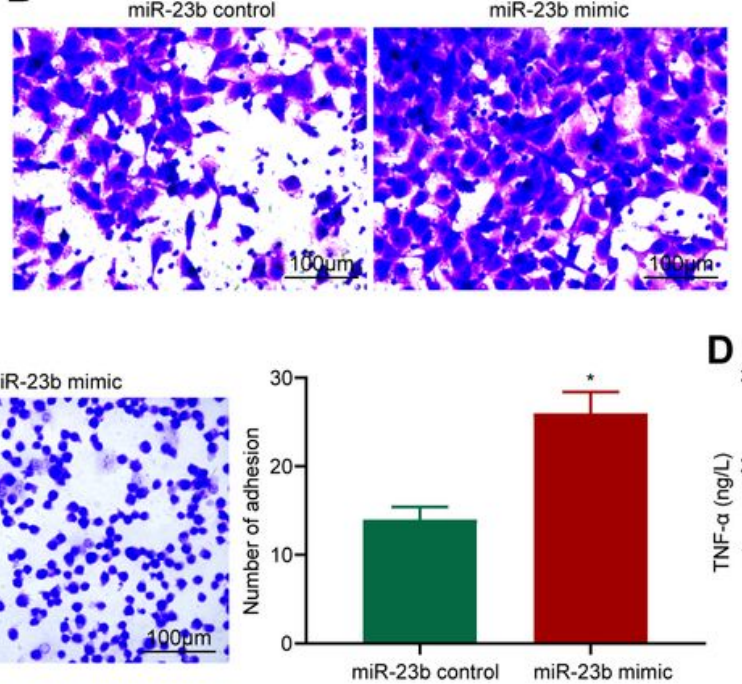

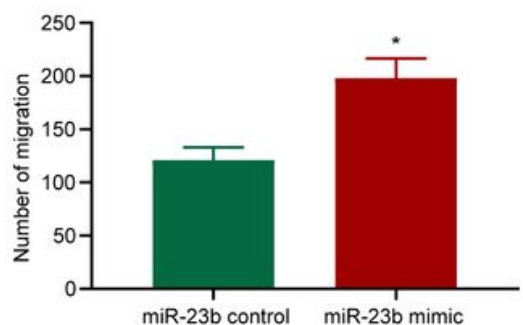

D

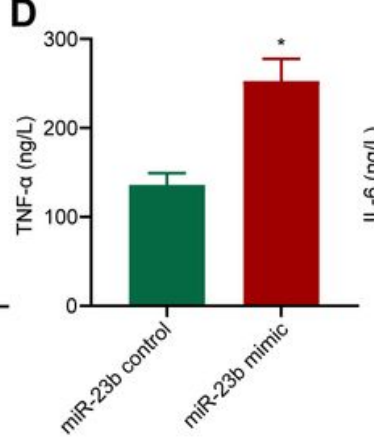

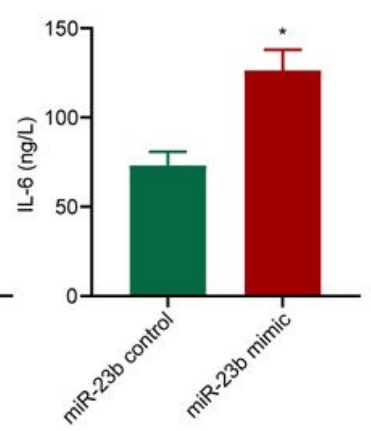

Figure 4

Effect of miR-23b mimic on macrophage. A, miR-23b expression in macrophage treated with miR-23b mimic detected by RT-qPCR ( ${ }^{\star} p<0.05$ according to the unpaired $t$ test). $B$, migration ability in macrophage treated with miR-23b mimic detected by Transwell assay $\left({ }^{\star} p<0.05\right.$ according to the unpaired $t$ test). $C$, cell adhesion ability in macrophage treated with miR-23b mimic detected by cell adhesion assay ( ${ }^{\star} p<0.05$ according to the unpaired $t$ test). D, TNF-a and IL- 6 levels in mouse aortic plasma detected by ELSA ( ${ }^{*} p<0.05$ according to the unpaired $t$ test). All experiment was conducted 3 times independently. 

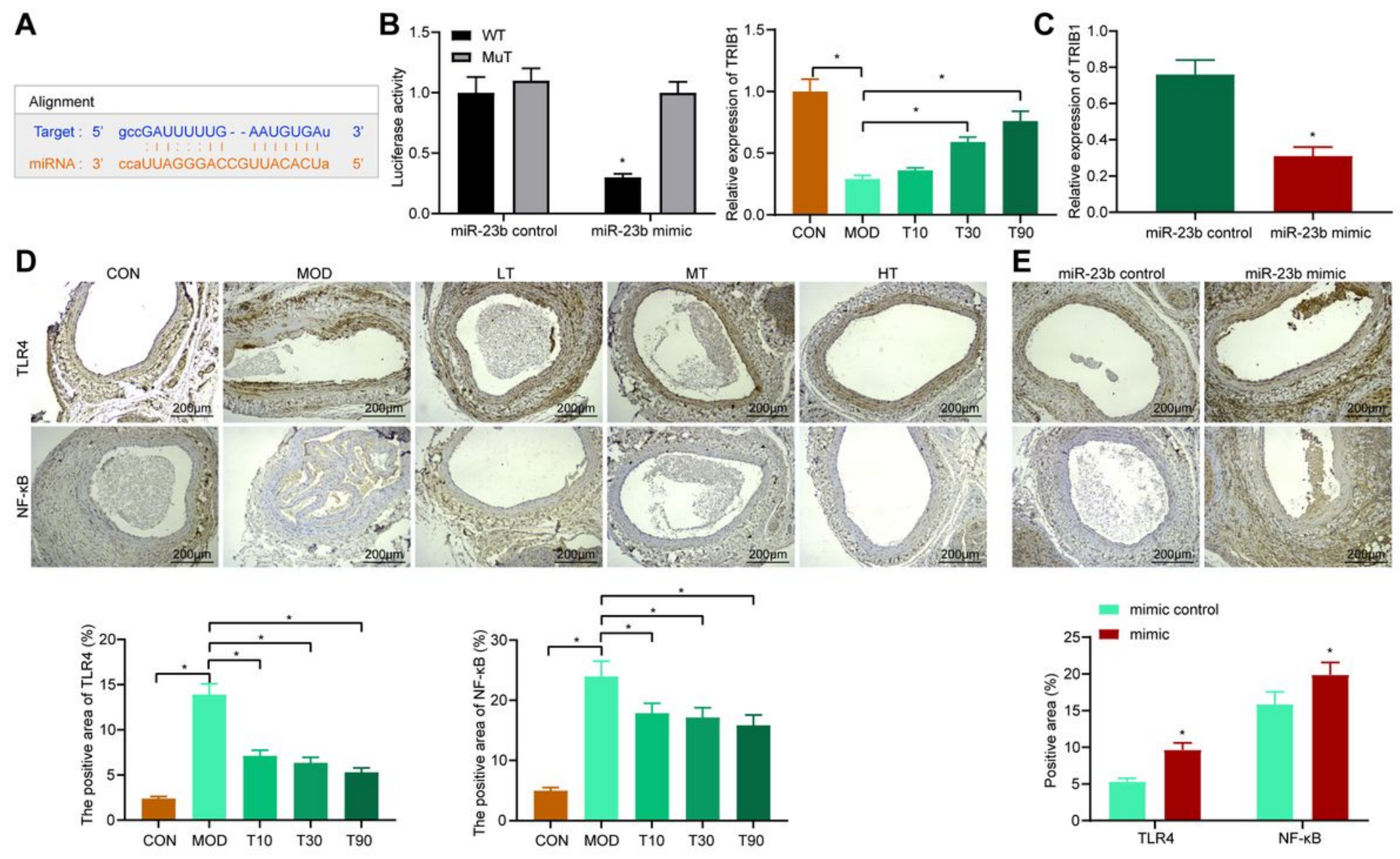

\section{Figure 5}

miR-23b regulates TRIB1 and the TLR4/NF-KB signaling pathway. A, mRNA target of miR-23b predicted by StarBase website and target relationship between miR-23b and TRIB1 verified by dual luciferase reporter gene assay ( ${ }^{*} p<0.05$ according to the two-way ANOVA). B and C, TRIB1 expression detected by RT-qPCR ( ${ }^{*} p<0.05$ according to the one-way ANOVA). D and E, expression of TLR4 and NF-KB detected by immunohistochemistry ( ${ }^{*} \mathrm{p}<0.05$ according to the two-way ANOVA). All experiment was conducted 3 times independently. 


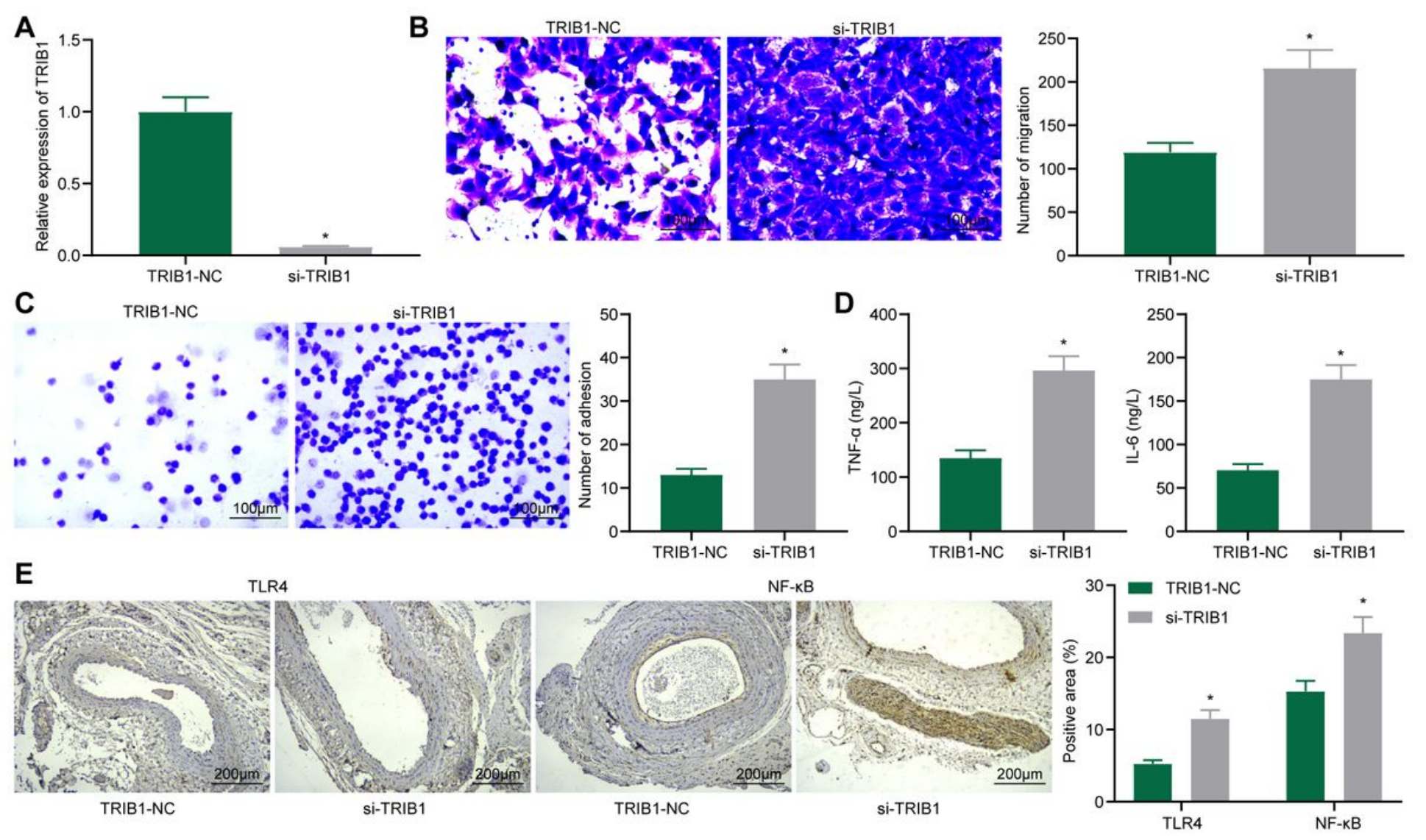

Figure 6

Effect of si-TRIB1 on macrophage. A, TRIB1 expression in macrophage treated with si-TRIB1 detected by RT-qPCR ( ${ }^{\star} p<0.05$ according to the unpaired $t$ test). B, migration ability in macrophage treated with siTRIB1 detected by Transwell assay ( ${ }^{*} p<0.05$ according to the unpaired $t$ test). $C$, cell adhesion ability in macrophage treated with si-TRIB1 detected by cell adhesion assay $\left({ }^{*} \mathrm{p}<0.05\right.$ according to the unpaired $\mathrm{t}$ test). D, TNF- $a$ and IL- 6 levels in mouse aortic plasma detected by ELSA ( ${ }^{*} p<0.05$ according to the unpaired $t$ test). $E$, TLR4/NF-KB activity in macrophage detected by immunohistochemistry $\left({ }^{*} p<0.05\right.$ according to the two-way ANOVA). All experiment was conducted 3 times independently. 

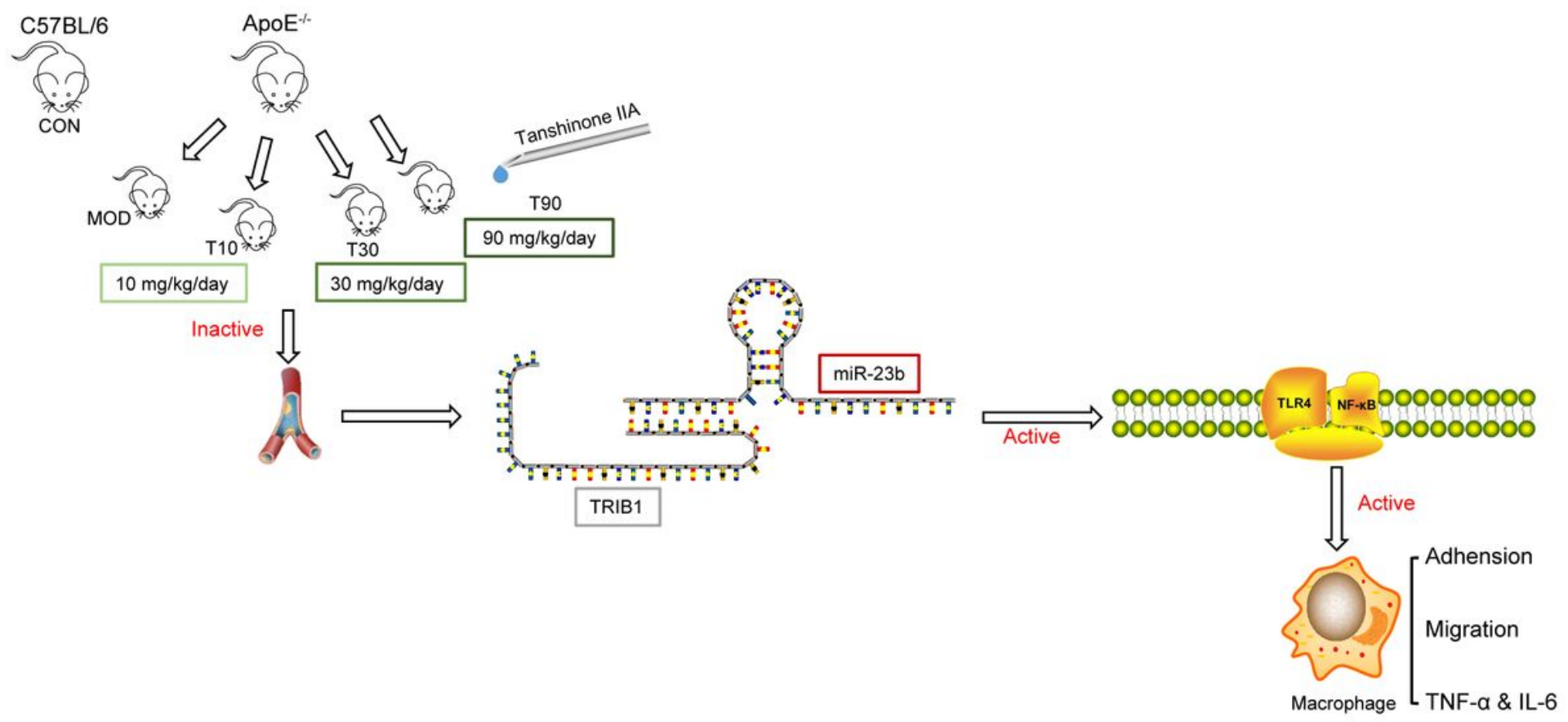

\section{Figure 7}

Mechanism of Tan, miR-23b, TRIB1 and TLR4/NF-KB in macrophage of atherosclerosis. Different concentrations of Tan treated mice were used to detect the atherosclerotic area of the aorta. miR-23b exerts an inhibitory function on Tan effect, and negatively targets TRIB1 to enhanced the activity of TLR4/NF-KB signaling pathway, so as to enhance the migration, adhesion ability and inflammatory reaction of macrophages.

\section{Supplementary Files}

This is a list of supplementary files associated with this preprint. Click to download.

- BCARD2000506.zip

- ARRIVEChecklist.docx 